\title{
French and German Abstracts
}

\author{
Social continuity and religious coexistence: the Muslim community of Tudela in \\ Navarre before the expulsion of 1516 \\ Carlos Conde Solares, University of Northumbria, Newcastle upon Tyne
}

Continuité sociale et coexistence religieuse: la communauté musulmane de Tudela en Navarre, avant l'expulsion de 1516

Cet article évalue la présence de communautés musulmanes dans le royaume de Navarre à la fin du Moyen Age. Après la reconquête chrétienne de la rive navarraise de l'Ebre en 1119, une importante communauté musulmane est restée en territoire chrétien jusqu'en 1516. L'étude se concentre sur le XVe siècle, une période pour laquelle la coexistence religieuse dans le plus petit des royaumes ibériques chrétiens appelle une mise en contexte. Une analyse des travaux existants alliée à de nouvelles données d'archives éclaire d'un jour nouveau les activités économiques des musulmans de Tudela ainsi que leurs relations avec la monarchie navarraise. Nous mettons en lumière leur identité collective, leurs systèmes juridiques, leurs relations non seulement avec leurs voisins chrétiens et juifs, mais aussi avec les autres communautés musulmanes ibériques, y compris celles d'Andalousie ou d'Ibérie maure.

Soziale Kontinuität und religiöse Koexistenz: die muslimische Gemeinde in Tudela in Navarra vor der Vertreibung von 1516

Dieser Beitrag fragt nach der Bedeutung muslimischer Gemeinden im Königreich Navarra im Sptätmittelalter. Im Anschluss an die christliche Rückeroberung Navarras nördlich des Ebro im Jahre 1119 gab es bis 1516 eine beträchtliche muslimische Gemeinschaft auf christlichem Territorium. Dieser Beitrag konzentriert sich auf das 15. Jahrhundert, denn in diesem Zeitraum herrschte in diesem 
kleinsten der christlichen Königreiche auf der Iberischen Halbinsel religiöse Toleranz, die allerdings einer weiteren Kontextualisierung bedarf. Ausgehend vom gegenwärtigen Forschungsstand wie auch von neuem Archivmaterial beleuchtet er die Wirtschaftstätigkeit der Moslems in Tudela, ihre Beziehungen mit dem Königshaus Navarra, ihre kollektive Identität, ihr Rechtssystem sowie die Beziehungen nicht nur zu ihren christlichen und jüdischen Nachbarn, sondern auch zu anderen iberischen muslimischen Gemeinschaften, einschließlich derjenigen in Al-Andalus, dem maurischen Iberien.

\section{Floods and money: funding drainage and flood control in coastal Flanders from the thirteenth to the sixteenth centuries Tim Soens, University of Antwerp, Belgium}

Inondations et gros sous: financement du drainage et prévention des inondations en Flandre côtière du XIIIe au XVIe siècle

Depuis le Haut Moyen Âge, les zones côtières de la mer du Nord ont été constamment regagnées et occupées. Afin de permettre une production agricole intensive dans ces secteurs, un système de drainage complexe a été progressivement installé, accompagné d'un contrôle des inondations. Ce système exigeait un investissement permanent en capitaux colossaux et en travail. Comme l'entretien du système de contrôle de l'eau était vital pour le fonctionnement du mode de production agricole côtier, l'évolution à long terme des investissements est un indicateur important, bien que rarement utilisé, de la situation économique, sociale et environnementale des zones du littoral. Le présent article repose sur des séries statistiques encore inédites et commençant fort tôt, concernant le financement de la maîtrise de l'eau en Flandre au Bas Moyen Age. Il montre que les fluctuations à long terme dans le financement du drainage et de la lutte contre les inondations ont été d'abord, et avant tout, liées à des changements structurels dans l'économie du littoral: une diminution globale des niveaux d'investissement se produisit en parallèle à la crise du XIVe siècle qui frappa, dans cette région, l'économie des petits paysans. Par contre, les contraintes exogènes, comme les grandes tempêtes, ne provoquaient qu'une perturbation à court terme des investissements.

Flut und Geld: die Finanzierung der Entwässerung und Flutkontrolle in den Küstenregionen Flanderns vom 13. bis zum 16. Jahrhundert

Die Feuchtgebiete an der Nordseeküste sind vom Hochmittelalter an in großem Umfang trockengelegt und besiedelt worden. Um in diesen Regionen eine intensive landwirtschaftliche Produktion zu ermöglichen, wurde nach und nach ein komplexes Entwässerungs- und Flutkontrollsystem aufgebaut, das ständige und umfangreiche Investitionen an Kapital und Arbeitskraft erforderte. Da die 
Instandhaltung des Wasserkontrollsystems für das Agrarsystem der Küstenregion geradezu lebensnotwendig war, stellt die langfristige Entwicklung der Investitionen einen wichtigen - wenn auch selten genutzten - Indikator für die ökonomischen, sozialen und umweltbedingten Geschicke der Küstenregionen dar. Auf der Basis neuer und sehr früher serieller Daten zur Finanzierung der Wasserkontrolle im spätmittelalterlichen Flandern vertritt dieser Beitrag die Auffassung, dass die langfristigen Fluktuationen der Aufwendungen für Entwässerung und Flutkontrolle in allererster Linie von den strukturellen Veränderungen innerhalb der Küstenwirtschaft beeinflusst wurden. Dabei verlief der Rückgang der Investitionshöhe parallel zur Krise der kleinbäuerlichen Wirtschaft dieser Region im 14. Jahrhundert, während exogene Belastungen wie z.B. Sturmfluten nur kurzfristige Unterbrechungen der Investitionen hervorriefen.

\section{Social mobility and the Middle Ages \\ Sandro Carocci, Università di Roma Tor Vergata, Dipartimento di Storia, Rome, Italy}

Mobilité Sociale et Moyen Âge

Malgré sa pertinence, la mobilité sociale n'a pas été une préoccupation majeure pour les médiévistes. La première partie de cet article traite des raisons pouvant expliquer ce manque d'intérêt, en soulignant le rôle qu'ont pu jouer certains modèles historiques, comme la «révolution féodale» mise en avant par les historiens français, les interprétations néo-malthusiennes, le modèle de commercialisation défini par les Anglais, et la grande épopée des marchands italiens. La seconde partie évalue dans quelle mesure ce manque d'intérêt des médiévistes pour la mobilité sociale a été contrebalancé par de nouvelles conceptions de l'espace social et les schémas de mobilité sociale mis au point et défendus, dans les dernières décennies, par sociologues et anthropologues. Par conséquent, il est vraiment important d'indiquer les lacunes de notre compréhension, et de clarifier la problématique de recherche, les questions techniques et méthodologiques. Cet essai définit les éléments constitutifs des identités sociales, la pluralité des échelles sociales, et les canaux de mobilité sociale. Il aborde le rôle modélisant des représentations acquises, et les contraintes imposées sur l'action des individus par les usages familiaux et le genre. Il souligne l'importance d'étudier la mobilité à l'intérieur des groupes sociaux, et fait valoir qu'il faut bien distinguer entre deux types de mobilité sociale à l'époque médiévale: d'une part une mobilité sociale «auto-générée» et d'autre part une mobilité sociale endogène, créatrice de conflits.

\section{Soziale Mobilität und das Mittelalter}

Ihrer großen Bedeutung zum Trotz gehört die soziale Mobilität nicht zu den bevorzugten Gegenständen der Historiker des Mittelalters. Der erste Teil dieses 
Beitrags beschäftigt sich mit den Gründen für dieses mangelnde Interesse und beleuchtet dabei vor allem die Rolle historischer Modelle wie die französische „Feudalrevolution“, neo-malthusianische Interpretationen, das englische Kommerzialisierungsmodell und die Großen Erzählung der italienischen Fernhändler des Mittelalters. Der zweite Teil untersucht, in welchem Maße das besagte Desinteresse durch die Konzepte der sozialen Raums und der sozialen Mobilität, wie sie in den letzten Jahrzehnten von Soziologen und Anthropologen entwickelt worden sind, herausgefordert wird. Es ist daher wirklich entscheidend, die Lücken unseres Verständnisses zu benennen sowie Forschungsfragen, technische Probleme und Methoden zu klären. Der Beitrag untersucht die konstitutiven Elemente sozialer Identitäten, die Vielfalt sozialer Leitern und die Kanäle der sozialen Mobilität. Er behandelt die performative Rolle gelehrter Repräsentationen und die Einschränkungen menschlichen Handelns durch Familienpraktiken. Er unterstreicht die Bedeutung des Studiums der Mobilität innerhalb sozialer Gruppen und behauptet, dass wir im Mittelalter zwei unterschiedliche Typen der sozialen Mobilität unterscheiden müssen: zum einen die eigenständige und zum andern die endogene oder konfliktbezogene soziale Mobilität.

\section{Bread provisioning and retail dynamics in the southern Low Countries: the bakers of Leuven, 1600-1800 \\ Brecht Dewilde and Johan Poukens, Early Modern History Research Unit, University of Leuven}

Ravitaillement en pain et dynamique de vente au détail dans les Pays-Bas méridionaux: les boulangers de Louvain, 1600-1800

L'argument central de cet article est que, jusqu'à présent, la recherche historique concernant la croissance du commerce de détail à l'époque moderne et ses méthodes s'est beaucoup trop concentrée sur l'étude de la vente au détail de biens durables (à l'exception peut-être de ce qui touche les épices coloniales) et sur les corporations correspondantes de marchands détaillants. Ainsi, le rôle des corporations qui regroupaient les producteurs alimentaires et les marchands détaillants, selon leur métier, n'a pas encore reçu suffisamment d'attention. La recherche sur les pratiques de vente au détail n'a pas été reliée à un courant ancien de la recherche historique, pourtant toujours vivant, qui voyait les historiens s'intéresser essentiellement aux niveaux de consommation des denrées alimentaires de base. A notre avis, si l'on aborde le fait de vendre du pain comme un «système d'approvisionnement», alors les circuits de vente au détail qui sont en concurrence et les différentes façons dont les crises de subsistance ultérieures ont pu affecter chacun de ces circuits permettent de proposer une explication supplémentaire (au-delà des changements bien connus et documentés de la demande) justifiant d'inclure d'autres maîtres de métier regroupés en corporations - en l'occurrence les boulangers de Louvain - au sein de la liste des maîtres de métiers concernant les produits de première nécessité, au même titre que les épiciers vendant des produits 
coloniaux, les vendeurs de vêtements et autres biens durables, les inscrivant tous dans l'éventail des maîtres producteurs, organisés en corporations, grands et petits marchands de produits alimentaires traditionnels.

Brotversorgung und Einzelhandelsdynamik in den südlichen Niederlanden: die Bäcker von Leuven, 1600-1800

Die zentrale These dieses Beitrags lautet, dass die gegenwärtige historische Forschung zum Wachstum und zu den Praktiken des frühneuzeitlichen Einzelhandels sich zu eng auf den Einzelhandel von Gebrauchsgütern (vielleicht mit Ausnahme von Lebensmitteln aus den Kolonien) und auf Einzelhändlerzünfte und -gilden konzentriert hat, während die Herstellung und der Verkauf von Nahrungsmitteln und/oder die Zünfte in diesem Bereich bisher weit weniger Beachtung gefunden haben. Die Forschung zu den Praktiken des Einzelhandels hat noch keine Verbindung zu jener älteren (und noch immer lebhaften) Forschungstradition gefunden, die sich in der Hauptsache mit dem Konsum von Grundnahrungsmitteln beschäftigt hat. Wenn man den Brotverkauf als „Versorgungssystem“ versteht, dann - so unsere These - bieten konkurrierende Einzelhandelskreise und die unterschiedliche Art und Weise, in der anschließende Subsistenzkrisen jeden dieser Kreise beeinflussten, eine zusätzliche Erklärung (neben den gut dokumentierten Nachfrageveränderungen) dafür an, warum auch andere Grundnahrungsmittel, koloniale Lebensmittel, Kleidung und selbst langlebige Konsumgüter ins Sortiment der traditionellen, im Bereich der Nahrungsmittelherstellung und des Nahrungsmitteleinzelhandels tätigen Zunftmeister - in diesem Fall: der Leuvener Bäcker - aufgenommen wurden.

\section{Making crime pay in late eighteenth-century Bristol: stolen goods, the informal economy and the negotiation of risk} Matt Neale, Centre for Urban History, University of Leicester

Pour que le crime paye à Bristol, vers la fin du XVIIIe siècle: marchandises volées, économie informelle et risque négocié

Cet article examine comment des biens volés ont été vendus et ont pu circuler de diverses façons, à la fin du XVIIIe siècle, à Bristol. C'est à juste titre qu'on a rapproché l'importance du commerce de seconde main et la vitalité de «l'économie informelle», dans cette période, de la fréquente mise sur le marché de biens volés, mais l'exemple que nous apportons de la ville de Bristol remet en cause un certain nombre d'interprétations et de théories antérieurement soutenues par les historiens sur le fonctionnement du marché des biens volés à cette époque. On peut donc se demander dans quelle mesure des modèles de criminalité, reposant sur l'exemple de Londres, peuvent être appliqués aux villes de province en Angleterre. L'article attire également l'attention sur les contraintes que devaient subir les voleurs avant 
de pouvoir écouler les marchandises qu'ils avaient volées: pour les mettre sur le marché, ils devaient tenir compte de facteurs les concernant tels que le calendrier, l'apparence et le lieu.

Damit das Verbrechen sich auszahlt: gestohlene Güter, informelle Ökonomie und Risikostreuung in Bristol im 18. Jahrhundert

Dieser Beitrag untersucht die vielfältige Weise, in der gestohlene Güter im späten 18. Jahrhundert in Bristol verkauft wurden und zirkulierten. Auch wenn Historiker zu Recht auf darauf hingewiesen haben, wie wichtig damals der Gebrauchtwarenmarkt und die ,,informelle Ökonomie“ für den Absatz gestohlener Güter war, werden dennoch - so die These - einige der Beschreibungen und Modelle der Märkte für Diebesgut durch das Quellenmaterial aus Bristol nur zum Teil bestätigt. Dies wirft die Frage auf, inwiefern Kriminalitätsmodelle, die sich auf London beziehen, auf englische Provinzstädte übertragen werden können. Der Beitrag richtet sein Augenmerk auch darauf, dass die Fähigkeit der Diebe, ihr Diebesgut zu verkaufen, durch verschiedene Faktoren wie z.B. Ort, Zeit und äußeres Erscheinungsbild beeinträchtigt wurde. 